\title{
Efficient Edelstein effects in one-atom-layer Tl-Pb compound
}

\author{
Y. Shiomi ${ }^{1}$ K. T. Yamamoto ${ }^{1}$, R. Nakanishi ${ }^{2}$, T. Nakamura ${ }^{2}$, \\ S. Ichinokura ${ }^{2}$, R. Akiyama ${ }^{2}$, S. Hasegawa ${ }^{2}$, and E. Saitoh ${ }^{1,3,4,5}$ \\ 1 Institute for Materials Research, Tohoku University, Sendai 980-8577, Japan \\ 2 Department of Physics, University of Tokyo, Tokyo 113-0033, Japan \\ 3 Advanced Institute for Materials Research, Tohoku University, Sendai 980-8577, Japan \\ 4 Advanced Science Research Center, Japan Atomic Energy Agency, Tokai 319-1195, Japan and \\ ${ }^{5}$ Center for Spintronics Research Network, Tohoku University, Sendai 980-8577, Japan
}

(Dated: March 7, 2022)

\begin{abstract}
We have investigated direct and inverse Edelstein effects in a one-atom-layer $\mathrm{Tl}-\mathrm{Pb}$ compound with a large Rashba-type spin splitting. In spin pumping experiments at room temperature, spinto-charge conversion voltage due to the inverse Edelstein effect is clearly observed in $\mathrm{Py} / \mathrm{Cu} / \mathrm{Tl}-\mathrm{Pb}$ trilayer samples. To confirm efficient spin-charge interconversion in $\mathrm{Tl}-\mathrm{Pb}$ compounds, the direct Edelstein effect is also studied in the same trilayer samples by measuring modulation of the effective magnetization damping in the Py layer via the charge-to-spin conversion in the Tl-Pb layer. Using both the results of direct and inverse Edelstein effects, the Edelstein length is estimated to be $\sim 0.1$ $\mathrm{nm}$ for $\mathrm{Tl}-\mathrm{Pb}$ compounds.
\end{abstract}

Two-dimensional electronic states have recently attracted much attention in the spintronics field [1]. Spaceinversion asymmetry leads to spin splitting of twodimensional electronic states due to the Rashba-type spin-orbit interaction [2-4]. The spin split bands are characterized by Fermi contours with helical locking of spin with momentum. The helical spin texture accompanies nonzero spin accumulation along an inplane direction transverse to the direction of applied electric currents, which is known as an Edelstein effect [5]. As a spin-to-charge conversion effect in the two-dimensional systems, an inverse effect of the Edelstein effect, the inverse Edelstein effect [6], has been studied by using the spin pumping technique on the surface of topological insulators $7-13]$ and the interface of bilayer materials 6, 14 16]. Since the Edelstein effects on two-dimensional Rashba states have potentially better efficiencies of spincharge interconversion than the inverse spin Hall effect observed in conventional heavy metals [17, 18], material search for the efficient Edelstein effects could be important for future spintronics applications.

Atomic-layer metal films on Si (silicon) substrates have been intensively studied in the field of surface science for more than 50 years [19]. Because of the surface reconstruction on $\mathrm{Si}$, surface superstructures can possess conducting surface states, which are inherently two dimensional and decoupled from the bulk. Although conductivity of ultrathin films is usually suppressed by reducing the film thickness, monolayers of In- and Pb-induced surface superstructures on Si (111) have good conductivity and even show superconductivity at low temperatures (1-

\footnotetext{
* Present address: Department of Applied Physics and QuantumPhase Electronics Center (QPEC), University of Tokyo, Hongo, Tokyo 113-8656, Japan, and RIKEN Center for Emergent Matter Science (CEMS), Wako 351-0198, Japan

${ }^{\dagger}$ Present address: Department of Applied Physics, University of Tokyo, Hongo, Tokyo 113-8656, Japan
}

\section{$3 \mathrm{~K})[20$ 28].}

Recently, it was reported that alloying one monolayer of $\mathrm{Tl}$ with one-third monolayer of $\mathrm{Pb}$ results in a oneatom-layer $\mathrm{Tl}-\mathrm{Pb}$ compound on $\mathrm{Si}$ (111) that exhibits both two-dimensional superconductivity $(2.25 \mathrm{~K})$ and Rashba-type spin splitting 29]. Angle-resolved photoelectron spectroscopy showed that the magnitude of the Rashba-type spin splitting reaches $\sim 250 \mathrm{meV}$ [29], which is even larger than that in one-third monolayer of $\mathrm{Bi}$ on $\mathrm{Ag}$ surface alloys $(\sim 200 \mathrm{meV})$ [30]. Although giant Rashba-type spin splitting has been also observed in Bi 31 33 and Tl 34 38 monolayers on Si (111) surfaces, these systems are non-metallic, not suitable for spintronics applications. Hence, the $\mathrm{Tl}-\mathrm{Pb}$ compounds on $\mathrm{Si}$ are rare atomic-layer materials promising for spin-charge interconversion due to the Edelstein effects.

In this manuscript, we report spin-charge interconversion effects in $\mathrm{Tl}-\mathrm{Pb}$ compounds. By spin pumping from a ferromagnetic permalloy ( $\mathrm{Py}$ ) layer to the $\mathrm{Tl}-\mathrm{Pb}$ layer, large Lorentz-type voltage peaks were observed at ferromagnetic resonance (FMR) of $\mathrm{Py}$, while small antisymmetric voltage signals due to ferromagnetic transport was observed only in a control sample without the $\mathrm{Tl}-\mathrm{Pb}$ compound. The dominant Lorentz-type voltage signal induced by spin pumping into the $\mathrm{Tl}-\mathrm{Pb}$ layer strongly indicates that the inverse Edelstein effect emerges on the Tl$\mathrm{Pb}$ compound. Furthermore, to evaluate the efficiency of the Edelstein effects in the $\mathrm{Tl}-\mathrm{Pb}$ compound reliably, we also measured the magnetization-damping modulation of the Py layer induced by spin transfer torque originating from the Edelstein effect on the Tl-Pb layer. From the experimental results of the Edelstein effect and the inverse Edelstein effect, the Edelstein length [6], which is a measure of the spin-charge conversion efficiency, was estimated to be $\sim 0.1 \mathrm{~nm}$ for $\mathrm{Tl}-\mathrm{Pb}$; this magnitude is comparable to that reported in $\mathrm{Py} /$ topological-insulator samples, e.g. $\mathrm{Py} / \mathrm{Bi}_{2} \mathrm{Se}_{3}$ [8] and $\mathrm{Py} / \mathrm{Cu} / \mathrm{Sn}-\mathrm{Bi}_{2} \mathrm{Te}_{2} \mathrm{Se}$ [11].

Atomic-layer $\mathrm{Tl}-\mathrm{Pb}$ compounds were prepared by a molecular beam epitaxy method, following the process 


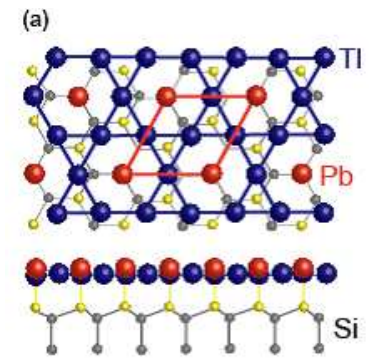

(b)
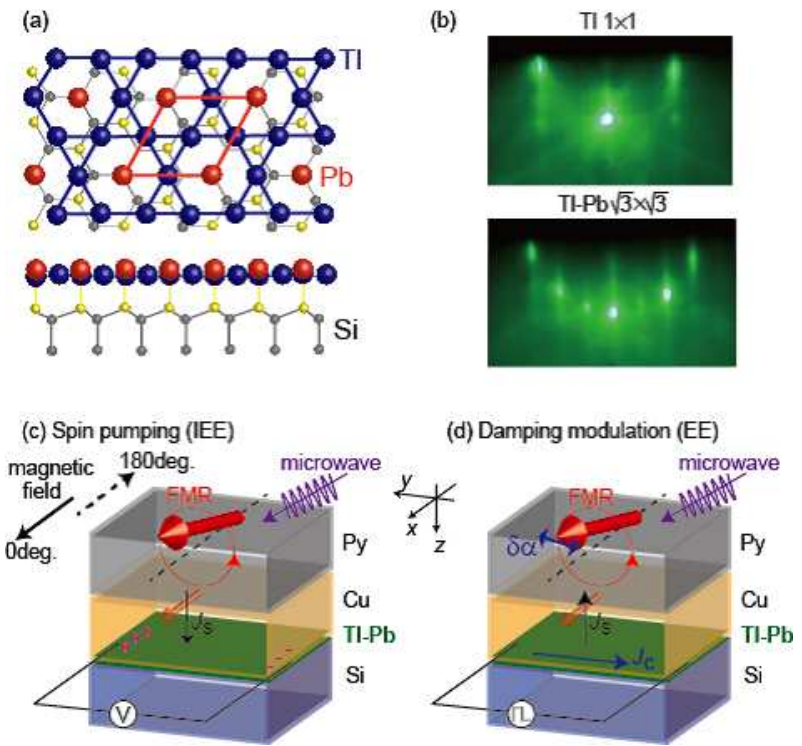

FIG. 1: (Color online.) (a) Atomic structure of the Tl-Pb compound grown on a $\mathrm{Si}(111)$ substrate. $\mathrm{Tl}$ and $\mathrm{Pb}$ atoms are shown by blue and red circles, respectively. Si atoms are also shown by yellow and gray circles. The $\sqrt{3} \times \sqrt{3}$ unit cell is outlined by a red frame. (b) In situ RHEED patterns during the growth process of a Tl-Pb compound. (c) Schematic illustration of the inverse Edelstein effect (IEE) induced by spin pumping into the $\mathrm{Tl}-\mathrm{Pb}$ layer. (d) Schematic illustration of modulation of the effective damping constant via spin transfer through the Edelstein effect (EE) in the Tl-Pb layer.

established by some of the present authors [29]. Pristine $\mathrm{Tl} / \mathrm{Si}(111)-(1 \times 1)$ reconstruction was made by depositing one-monolayer $\mathrm{Tl}$ onto a $\mathrm{Si}(111)-(7 \times 7)$ surface at $\sim 300{ }^{\circ} \mathrm{C}$. Then, one-third monolayer of $\mathrm{Pb}$ was deposited onto the $\mathrm{Tl} / \mathrm{Si}(111)$ surface at room temperature. The surface reconstruction was confirmed by monitoring reflection high-energy electron diffraction (RHEED) patterns during the growth process, as shown in Fig. 1(b). The RHEED pattern of the Tl-Pb compound differs from that of the parent $\mathrm{Tl}$ monolayer, and $\sqrt{3} \times \sqrt{3}$ periodicity appears. On top of the $\mathrm{Tl}-\mathrm{Pb}$ compounds prepared on the $\mathrm{Si}$ substrates, a 60 -nm-thick $\mathrm{Cu}$ capping layer was deposited with an in situ electron-beam evaporator. Since the spin diffusion length of $\mathrm{Cu}(\sim 500 \mathrm{~nm}[39,40])$ is much longer than the thickness of the $\mathrm{Cu}$ layer, the loss of spin current in the $\mathrm{Cu}$ layer is negligible. The $\mathrm{Cu} / \mathrm{Tl}-$ $\mathrm{Pb}$ bilayers were transferred to another vacuum chamber, and then a 20 -nm-thick Py $\left(\mathrm{Ni}_{81} \mathrm{Fe}_{19}\right)$ film was deposited onto them using electron beam evaporation to study the Edelstein effects in $\mathrm{Py} / \mathrm{Cu} / \mathrm{Tl}-\mathrm{Pb}$ trilayer samples.

First, we investigated the inverse Edelstein effect induced by spin pumping into the Tl-Pb layer. The experiments were performed at room temperature using an electron spin resonance (ESR) spectrometer [41], as illustrated in Fig. 1(c). $\mathrm{Py} / \mathrm{Cu} / \mathrm{Tl}-\mathrm{Pb}$ trilayer samples were placed in the center of the ESR cavity and a $9.4 \mathrm{GHz}$ microwave was applied to the samples. When an exter-
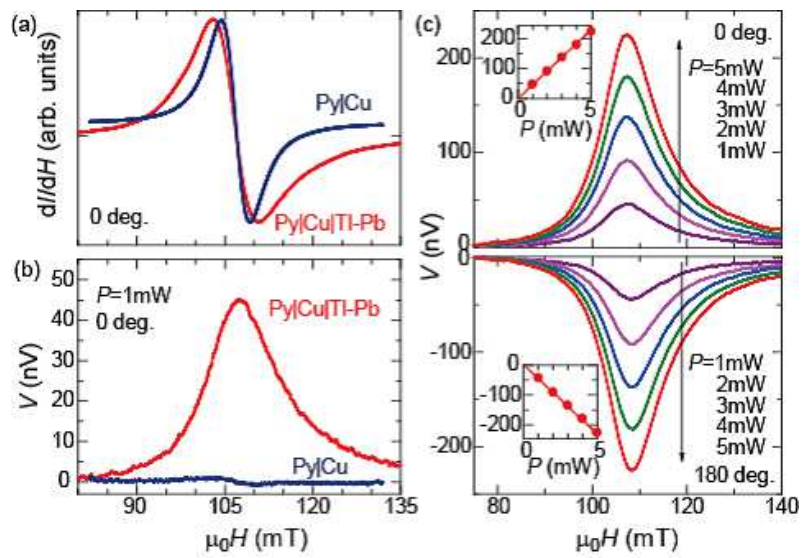

FIG. 2: (Color online.) (a) FMR derivative absorption spectra $(\mathrm{d} I / \mathrm{d} H)$ for $\mathrm{Py} / \mathrm{Cu} / \mathrm{Tl}-\mathrm{Pb}$ and $\mathrm{Py} / \mathrm{Cu}$ films. (b) Voltage spectra for $\mathrm{Py} / \mathrm{Cu} / \mathrm{Tl}-\mathrm{Pb}$ and $\mathrm{Py} / \mathrm{Cu}$ films. (c) Voltage spectra for $\mathrm{Py} / \mathrm{Cu} / \mathrm{Tl}-\mathrm{Pb}$ at various input-microwave power $(P)$ levels and different magnetic-field directions $(0 \mathrm{deg}$. and 180 deg.). The insets show the $P$ dependence of the voltage peak magnitudes at $0 \mathrm{deg}$. and $180 \mathrm{deg}$.

nal magnetic field applied along the film plane $[x$ axis in Fig. 1(c)] satisfies the FMR condition, the injection of spin currents into the $\mathrm{Tl}-\mathrm{Pb}$ layer takes place via the spin pumping. DC voltages arising on the $\mathrm{Tl}-\mathrm{Pb}$ layer along the $y$ axis in Fig. 1(c) around the FMR magnetic fields were measured using a nanovoltmeter. The length between the voltage electrodes is $2 \mathrm{~mm}$, and the sample width is $1 \mathrm{~mm}$.

Figure 2(a) shows an FMR derivative absorption spectrum, $\mathrm{d} I / \mathrm{d} H$, for $\mathrm{Py} / \mathrm{Cu} / \mathrm{Tl}-\mathrm{Pb}$ and $\mathrm{Py} / \mathrm{Cu}$ samples. FMR of the Py layer is observed for both the samples at the magnetic field of $\sim 105 \mathrm{mT}$. The FMR linewidth is clearly enhanced for the $\mathrm{Py} / \mathrm{Cu} / \mathrm{Tl}-\mathrm{Pb}$ trilayer compared to the $\mathrm{Py} / \mathrm{Cu}$ bilayer which does not include the $\mathrm{Tl}-\mathrm{Pb}$ layer: peak-to-peak linewidth $\Delta H_{\mathrm{p}-\mathrm{p}}=7.86 \mathrm{mT}$ for the $\mathrm{Py} / \mathrm{Cu} / \mathrm{Tl}-\mathrm{Pb}$ trilayer and $\Delta H_{\mathrm{p}-\mathrm{p}}=4.88 \mathrm{mT}$ for the $\mathrm{Py} / \mathrm{Cu}$ bilayer. The enhancement of the FMR linewidth can be ascribed to the spin pumping; the transfer of spin currents from $\mathrm{Py}$ to the $\mathrm{Tl}-\mathrm{Pb}$ layer effectively increases the damping of the Py magnetization precession.

Voltage spectra measured for $\mathrm{Py} / \mathrm{Cu} / \mathrm{Tl}-\mathrm{Pb}$ and $\mathrm{Py} / \mathrm{Cu}$ samples at around FMR magnetic fields are shown in Fig. 2(b). Here, the input microwave power is set at $1 \mathrm{~mW}$. For the $\mathrm{Py} / \mathrm{Cu} / \mathrm{Tl}-\mathrm{Pb}$ trilayer, a positive voltage peak with the magnitude of $\sim 45 \mathrm{nV}$ is clearly observed at around the FMR magnetic-field, while no voltage peak is discerned for the $\mathrm{Py} / \mathrm{Cu}$ bilayer. The line shape of the small voltage signal observed in the $\mathrm{Py} / \mathrm{Cu}$ bilayer is similar to that of voltage induced by the anomalous Hall effect in the Py layer [41]. At FMR, the Py magnetization precession generates spin currents. The resulting spin accumulation on the Tl-Pb layer is converted to a charge current by the inverse Edelstein effect. The Lorentz-type voltage peak observed in the 

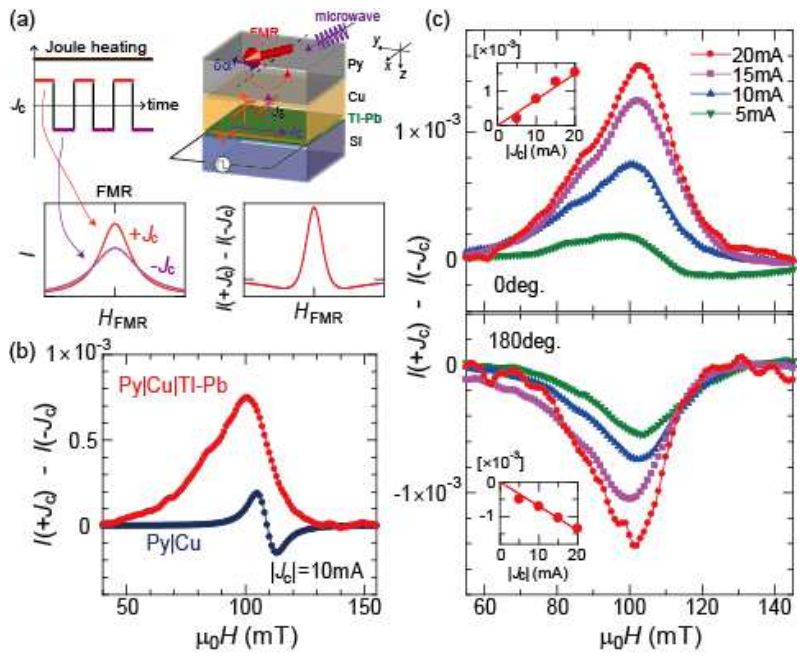

FIG. 3: (Color online.) (a) Schematic illustration of lock-in detection of the damping modulation, and predicted signal shapes of the experimental results. I denotes the FMR absorption intensity. (b) The modulation signal of the FMR spectrum as a function of an external magnetic field for $\mathrm{Py} / \mathrm{Cu} / \mathrm{Tl}-\mathrm{Pb}$ and $\mathrm{Py} / \mathrm{Cu}$. (c) The modulation signal at various electric-current $\left(J_{C}\right)$ magnitudes and at different magnetic-field directions ( 0 deg. and 180 deg.). The insets show the $\left|J_{C}\right|$ dependence of the modulation signals at $0 \mathrm{deg}$. and $180 \mathrm{deg}$.

$\mathrm{Py} / \mathrm{Cu} / \mathrm{Tl}-\mathrm{Pb}$ trilayer is consistent with the inverse Edelstein effect [6-16].

When the direction of the magnetic field is reversed from the $+x(0$ deg. $)$ to $-x(180$ deg. $)$ direction, the voltage peaks in the $\mathrm{Py} / \mathrm{Cu} / \mathrm{Tl}-\mathrm{Pb}$ sample change their sign as expected for the inverse Edelstein effect, as shown in Fig. 2(c). The magnitudes of the voltage peaks increase monotonically, as the input microwave power increases. As shown in the inset to Fig. 2(c), the microwave-power dependence is linear, which indicates that the observed spin pumping signals are in the linear regime.

The spin pumping signals in Fig. 2 allow us to estimate the Edelstein length [6], which is a measure of the conversion efficiency of the effect. From the FMR line widths shown in Fig. 2(a), the spin current injected into the $\mathrm{Tl}-\mathrm{Pb}$ layer is estimated to be $1592 \mathrm{~A} / \mathrm{m}^{2}$ using an established formula for spin pumping [41, 42]. Using the voltage magnitude shown in Fig. 2(b) and the sample resistance $(100 \Omega)$, the generated charge current is estimated to be $2.25 \times 10^{-7} \mathrm{~A} / \mathrm{m}$. Hence, the Edelstein length $\lambda_{E E}$ is obtained as the ratio of the charge to spin current: $0.14 \mathrm{~nm}$. This magnitude is comparable to the reported $\lambda_{E E}$ values for topological insulator $\mathrm{Bi}_{2} \mathrm{Se}_{3}(0.21 \mathrm{~nm})[8]$ and $\mathrm{Sn}-\mathrm{Bi}_{2} \mathrm{Te}_{2} \mathrm{Se}(0.27 \mathrm{~nm})$ [1] , but 15 times smaller than that for $\alpha$-Sn $(2.1 \mathrm{~nm})$ [10].

To confirm the efficient spin-charge interconversion in the $\mathrm{Tl}-\mathrm{Pb}$ compound, we next performed an experiment of the Edelstein effect in the same $\mathrm{Py} / \mathrm{Cu} / \mathrm{Tl}-\mathrm{Pb}$ trilayer sample, as shown in Fig. 3. The experimental setup for the detection of the Edelstein effect is illustrated in Fig. 1(d). When an electric current $J_{C}$ is applied to the $\mathrm{Tl}-\mathrm{Pb}$ layer along the $y$ axis, $\mathrm{Tl}-\mathrm{Pb}$ exhibits the Edelstein effect, and thus the charge current is converted to spin accumulation in the $\mathrm{Tl}-\mathrm{Pb}$ layer. The accumulated spin angular momentum is immediately transferred to the Py layer via a spin current propagating through the $\mathrm{Cu}$ layer along the $z$ axis. The magnetization-precession relaxation of the Py layer is modulated by the injected spin current, which can be detected as the modulation of the damping constant (line width) in the FMR spectra.

Several experiments of the damping modulation by spin transfer torque have been reported using the spin Hall effect in $\mathrm{Py} / \mathrm{Pt}$ systems [43, 44]. In these experiments, DC charge currents were applied to Pt layers, which may cause additional damping modulation due to heating effects [43]. To rule out extrinsic effects due to the heating effect, a lock-in detection is used in the present experiment, as shown in Fig. 3(a). When the direction of $J_{C}$ is reversed between $+y$ and $-y$ directions, the sign of the damping modulation induced by the Edelstein effect is reversed, whereas the heating effect does not change sign. Lock-in detection of the FMR spectra with the frequency of $J_{C}$ enables precise estimation of the damping modulation due to the Edelstein effect.

Figure 3(b) shows modulation of FMR absorption intensity $(I)$ by the $J_{C}$ directions, $I\left(+J_{C}\right)-I\left(-J_{C}\right)$, measured for the $\mathrm{Py} / \mathrm{Cu} / \mathrm{Tl}-\mathrm{Pb}$ trilayer and the $\mathrm{Py} / \mathrm{Cu}$ bilayer in the ESR cavity. Signals are observed around the FMR magnetic field of the Py layer for both the samples, but clearly, the observed signal shapes are different between $\mathrm{Py} / \mathrm{Cu} / \mathrm{Tl}-\mathrm{Pb}$ and $\mathrm{Py} / \mathrm{Cu}$. For the $\mathrm{Py} / \mathrm{Cu}$ bilayer, the signal shape is dispersion-type, and its magnitude is small relative to the $\mathrm{Py} / \mathrm{Cu} / \mathrm{Tl}-\mathrm{Pb}$ case. The dispersiontype signal may be explained by an alternate Oersted field caused by AC charge currents. In contrast, for the $\mathrm{Py} / \mathrm{Cu} / \mathrm{Tl}-\mathrm{Pb}$ trilayer, the signal shape is a peak, similar to the predicted signal shape for the Edelstein effect [Fig. [3(a)].

The modulation signal is further studied by changing the $J_{c}$ magnitudes and the magnetic-field directions $[0$ deg. ( $+x$ direction $)$ and 180 deg. ( $-x$ direction)] in Fig. [3(c). As $\left|J_{C}\right|$ increases from $5 \mathrm{~mA}$ to $20 \mathrm{~mA}$, the magnitudes of the peak signals monotonically increase. As shown in the insets to Fig. 3( (c), the $\left|J_{C}\right|$ dependence is linear, which rules out the heating-induced extrinsic effects [43]. Furthermore, when the direction of the magnetic field is reversed from the $+x$ to $-x$ direction, the peak signals keep their magnitude but change their sign. The results are consistent with the damping modulation due to the Edelstein effect.

Let us quantitatively estimate the Edelstein length from the modulation signal shown in Fig. 3, Following the theoretical formulation developed for the damping modulation due to the spin Hall effect [43], the change in the effective damping constant induced by the Edelstein 
effect $\Delta \alpha_{E E}$ is given by

$$
\Delta \alpha_{E E}=\frac{\gamma \mu_{0} \hbar}{A_{F} \lambda_{E E} \omega M e} J_{C} .
$$

Here, $\gamma$ is the gyromagnetic ratio, $A_{F}\left(=2 \times 10^{-11} \mathrm{~m}^{2}\right)$ is the cross-sectional area of the Py layer, $\omega$ is the angular frequency of the microwave $(9.4 \mathrm{GHz}), M(=0.745$ $\mathrm{T}$ [41]) is the Py magnetization, and the other symbols $\left(\mu_{0}, \hbar\right.$, and $e$ ) have their usual meanings. The full width at half maximum of the modulation signal $\Delta H$ in Fig. 3 corresponds to the difference in the damping modulation between $+J_{C}$ and $-J_{C}$ conditions: $(2 \omega / \gamma) 2 \Delta \alpha_{E E}$. Hence, using $\Delta H \sim 20 \mathrm{mT}$ at $\left|J_{C}\right|=10 \mathrm{~mA}$ in Fig. 3(b), we obtain $\lambda_{E E}=0.11 \mathrm{~nm}$ from eq. (1). The similar $\lambda_{E E}$ value to that obtained by the inverse Edelstein effect $(0.14 \mathrm{~nm})$ supports the efficient Edelstein effects in the $\mathrm{Tl}-\mathrm{Pb}$ compound.

In summary, we studied spin-charge interconversion effects in $\mathrm{Tl}-\mathrm{Pb}$ one-atom-layer material at room temperature. Two-dimensional $\mathrm{Tl}-\mathrm{Pb}$ compounds which consist of one monolayer of $\mathrm{Tl}$ and one-third monolayer of $\mathrm{Pb}$ were grown on $\mathrm{Si}$ (111) substrates by a molecular beam epitaxy method. By spin pumping from ferromagnetic permalloy films into the $\mathrm{Tl}-\mathrm{Pb}$ compounds, clear spincharge conversion voltage is observed, which can be ascribed to the inverse Edelstein effect due to the strong Rashba-type spin-orbit interaction of the $\mathrm{Tl}-\mathrm{Pb}$ layer. The measurement of the inverse effect of spin pumping, i.e. damping modulation by applied electric currents, confirms the high efficiency of the Edelstein effects in the $\mathrm{Tl}-\mathrm{Pb}$ compounds. From the results of the direct and inverse Edelstein effects in the $\mathrm{Py}|\mathrm{Cu}| \mathrm{Tl}-\mathrm{Pb}$ trilayers, the Edelstein length is estimated to be $0.11-0.14 \mathrm{~nm}$, which is comparable to that reported in the surfaces of topological insulators. The efficient Edelstein effects on $\mathrm{Si}$ substrates could be compatible with the semiconductor technology.

This research was supported by JST ERATO "Spin Quantum Rectification Project" (JPMJER1402), JSPS (KAKENHI No. 16H02108, No. JP16H00983, No. 17H04806, No. 18H04215, and No. $18 \mathrm{H} 04311$ and the Core-to-Core program "International research center for new-concept spintronics devices") and MEXT (Innovative Area "Nano Spin Conversion Science" (No. 26103005)).
[1] W. Han, APL Materials 4, 032401 (2016).

[2] M. S. Kushwaha, Phys. Rev. B 74, 045304 (2006).

[3] M. S. Kushwaha, Phys. Rev. B 76, 245315 (2007).

[4] M. S. Kushwaha, J. Appl. Phys. 104, 083714 (2008).

[5] V. M. Edelstein, Solid State Commun. 73, 233-235 (1990).

[6] J. C. Rojas-Sánchez, L. Vila, G. Desfonds, S. Gambarelli, J. P. Attané, J. M. De Teresa, C. Magén, and A. Fert, Nat. Commun. 4, 2944 (2013).

[7] Y. Shiomi, K. Nomura, Y. Kajiwara, K. Eto, M. Novak, Kouji Segawa, Yoichi Ando, and E. Saitoh, Phys. Rev. Lett. 113, 196601 (2014).

[8] P. Deorani, J. Son, K. Banerjee, N. Koirala, M. Brahlek, S. Oh, and H. Yang, Phys. Rev. B 90, 094403 (2014).

[9] M. Jamali, J. S. Lee, J. S. Jeong, F. Mahfouzi, Y. Lv, Z. Zhao, B. K. Nikolic, K. A. Mkhoyan, N. Samarth, and J.-P. Wang, Nano Lett. 15, 7126 (2015).

[10] J. C. Rojas-Sánchez, S. Oyarzun, Y. Fu, A. Marty, C. Vergnaud, S. Gambarelli, L. Vila, M. Jamet, Y. Ohtsubo, A. Taleb-Ibrahimi, P. Le Fevre, F. Bertran, N. Reyren, J.-M. George, and A. Fert, Phys. Rev. Lett. 116, 096602 (2016).

[11] K. T. Yamamoto, Y. Shiomi, Kouji Segawa, Yoichi Ando, and E. Saitoh, Phys. Rev. B 94, 024404 (2016).

[12] Hailong Wang, James Kally, Joon Sue Lee, Tao Liu, Houchen Chang, Danielle Reifsnyder Hickey, K. Andre Mkhoyan, Mingzhong Wu, Anthony Richardella, and Nitin Samarth, Phys. Rev. Lett. 117, 076601 (2016).

[13] Qi Song, Jian Mi, Dan Zhao, Tang Su, Wei Yuan, Wenyu Xing, Yangyang Chen, Tianyu Wang, Tao Wu, Xian Hui Chen, X. C. Xie, Chi Zhang, Jing Shi, and Wei Han, Nature Commun. 7, 13485 (2016).

[14] S. Karube, K. Kondou, and Y. Ohtani, Appl. Phys. Express 9, 033001 (2016).
[15] Hanshen Tsai, Shutaro Karube, Kouta Kondou, Naoya Yamaguchi, Fumiyuki Ishii, Yoshichika Otani, Sci. Rep. 8, 5564 (2018).

[16] Qi Song, Hongrui Zhang, Tang Su, Wei Yuan, Yangyang Chen, Wenyu Xing, Jing Shi, Jirong Sun, and Wei Han, Sci. Adv. 3, e1602312 (2017).

[17] A. R. Mellnik, J. S. Lee, A. Richardella, J. L. Grab, P. J. Mintun, M. H. Fischer, A. Vaezi, A. Manchon, E.-A. Kim, N. Samarth, and D. C. Ralph, Nature (London) 511, 449 (2014).

[18] Yabin Fan, Pramey Upadhyaya, Xufeng Kou, Murong Lang, So Takei, Zhenxing Wang, Jianshi Tang, Liang He, Li-Te Chang, Mohammad Montazeri, Guoqiang Yu, Wanjun Jiang, Tianxiao Nie, Robert N. Schwartz, Yaroslav Tserkovnyak, and Kang L. Wang, Nat. Mater. 13, 699 (2014).

[19] J. J. Lander, Surf. Sci. 1, 125 (1964).

[20] T. Zhang, P. Cheng, W. J. Li, Y. J. Sun, G. Wang, X. G. Zhu, K. He, L. Wang, X. Ma, and X. Chen et al., Nat. Phys. 6, 104 (2010).

[21] S. Qin, J. Kim, Q. Niu, and C. K. Shih, Science 324, 1314 (2009).

[22] T. Uchihashi, P. Mishra, M. Aono, and T. Nakayama, Phys. Rev. Lett. 107, 207001 (2013).

[23] M. Yamada, T. Hirahara, and S. Hasegawa, Phys. Rev. Lett. 110, 237001 (2013).

[24] T. Uchihashi, P. Mishra, and T. Nakayama, Nanoscale Res. Lett. 8, 167 (2013).

[25] S. Yoshizawa, H. Kim, T. Kawakami, Y. Nagai, T. Nakayama, X. Hu, Y. Hasegawa, and T. Uchihashi, Phys. Rev. Lett. 113, 247004 (2014).

[26] J. Noffsiger and M. L. Cohen, Solid State Commun. 151, 421 (2011).

[27] W. Zhao, Q. Wang, M. Liu, W. Zhang, Y. Wang, M. 
Chen, Y. Guo, K. He, X. Chen, and Y. Wang et al., Solid State Commun. 165, 59 (2013).

[28] C. Brun, T. Cren, V. Cherkez, F. Debontridder, S. Pons, D. Fokin, M. C. Tringides, S. Bozhko, L. B. Ioffe, and B. L. Altshuler, et al. Nat. Phys. 10, 444 (2014).

[29] A. V. Matetskiy, S. Ichinokura, L. V. Bondarenko, A. Y. Tupchaya, D. V. Gruznev, A. V. Zotov, A.?A. Saranin, R. Hobara, A. Takayama, and S. Hasegawa, Phys. Rev. Lett. 115, 147003 (2015).

[30] Christian R. Ast, Jurgen Henk, Arthur Ernst, Luca Moreschini, Mihaela C. Falub, Daniela Pacilé, Patrick Bruno, Klaus Kern, and Marco Grioni, Phys. Rev. Lett. 98, 186807 (2007).

[31] I. Gierz, T. Suzuki, E. Frantzeskakis, S. Pons, S. Ostanin, A. Ernst, J. Henk, M. Grioni, K. Kern, and C. R. Ast, Phys. Rev. Lett. 103, 046803 (2009).

[32] Kazuyuki Sakamoto, Haruya Kakuta, Katsuaki Sugawara, Koji Miyamoto, Akio Kimura, Takuya Kuzumaki, Nobuo Ueno, Emilia Annese, Jun Fujii, Ayaka Kodama, Tatsuya Shishidou, Hirofumi Namatame, Masaki Taniguchi, Takafumi Sato, Takashi Takahashi, and Tamio Oguchi, Phys. Rev. Lett. 103, 156801 (2009).

[33] E. Frantzeskakis, S. Pons, and M. Grioni, Phys. Rev. B 82, 085440 (2010).

[34] Kazuyuki Sakamoto, Tatsuki Oda, Akio Kimura, Koji Miyamoto, Masahito Tsujikawa, Ayako Imai, Nobuo Ueno, Hirofumi Namatame, Masaki Taniguchi, P. E. J. Eriksson, and R. I. G. Uhrberg, Phys. Rev. Lett. 102, 096805 (2009).
[35] J. Ibañez-Azpiroz, A. Eiguren and A. Bergara, Phys. Rev. B 84, 125435 (2011).

[36] Kazuyuki Sakamoto, Tae-Hwan Kim, Takuya Kuzumaki, Beate Muller, Yuta Yamamoto, Minoru Ohtaka, Jacek R. Osiecki, Koji Miyamoto, Yasuo Takeichi, Ayumi Harasawa, Sebastian D. Stolwijk, Anke B. Schmidt, Jun Fujii, R. I. G. Uhrberg, Markus Donath, Han Woong Yeom, and Tatsuki Oda, Nat. Commun. 4, 2073 (2013).

[37] S. D. Stolwijk, A. B. Schmidt, M. Donath, K. Sakamoto, and P. Krüger, Phys. Rev. Lett. 111, 176402 (2013).

[38] S. D. Stolwijk, K. Sakamoto, A. B. Schmidt, P. Krüger, and M. Donath, Phys. Rev. B 90, 161109 (2014).

[39] S. Yakata, Y. Ando, T. Miyazaki, and S. Mizukami, Jpn. J. Appl. Phys. 45, 3892-3895 (2006).

[40] J. Bass and W. P. Pratt Jr., J. Phys.: Condens. Matter 19183201 (2007).

[41] K. Ando, S. Takahashi, J. Ieda, Y. Kajiwara, H. Nakayama, T. Yoshino, K. Harii, Y. Fujikawa, M. Matsuo, S. Maekawa, and E. Saitoh, J. Appl. Phys. 109, 103913 (2011).

[42] R. Iguchi and E. Saitoh, J. Phys. Soc. Jpn. 86, 011003 (2017).

[43] K. Ando, S. Takahashi, K. Harii, K. Sasage, J. Ieda, S. Maekawa, and E. Saitoh, Phys. Rev. Lett. 101, 036601 (2008).

[44] S. Kasai, K. Kondou, H. Sukegawa, S. Mitani, K. Tsukagoshi, and Y. Ohtani, Appl. Phys. Lett. 104, 092408 (2014). 